\title{
Gas Temperature and Cu Vapor Density in an Impulse Discharge
}

\author{
Student Member Tatsuya Sakoda \\ Member Masahisa Otsubo \\ Member Chikahisa Honda \\ Member Hiroshi Nieda
}

\author{
(Kyushu University) \\ (Miyazaki University) \\ (Miyazaki University) \\ (Kumamoto Institute of Technology)
}

\begin{abstract}
The laser scattering techniques are very powerful for the diagnostics of impulse discharges. Characteristics of gas temperature, density of $\mathrm{Cu}$ atoms from electrodes and electron density in and near an impulse discharge channel at an air pressure of $66.7 \mathrm{hPa}$ were investigated. The gas temperature took much higher values in the constricted regions of the channel, especially in the two regions near the tips of anode and cathode along the positive column. At the transition from glow to arc, the gas temperature and the densities of $\mathrm{Cu}$ atoms and electrons in the discharge channel became high with a rise of discharge current, and reached maximum values of $4800 \mathrm{~K}, 9 \times 10^{18} \mathrm{~m}^{-3}$ and $8 \times 10^{23} \mathrm{~m}^{-3}$, respectively. Both the positions of peaks of the gas temperature and the density of $\mathrm{Cu}$ atoms moved quickly from the inside toward the outside of discharge channel; their moving velocities decreased from $1200 \mathrm{~m} / \mathrm{s}$ to $200 \mathrm{~m} / \mathrm{s}$ within $20 \mu \mathrm{s}$.
\end{abstract}

Key words: Laser scattering techniques, Impulse discharge, Glow-to-arc transition, CARS, LIF, Thomson scattering

\section{Introduction}

An impulse discharge in low pressure air has a simple discharge channel, its reproducibility and some discharge phases in the discharge process. By this reason, the impulse discharge is convenient to clarify transitional mechanism between discharge phases and characteristics of each discharge phase. "1) (2) Studying the behavior in the discharge process consisting of glow and arc as sparkover phase, it will be also useful to obtain conditions of a stable glow discharge such as an excitation source of discharge pumped gas lasers. ${ }^{(3)}$

On the other hand, we have proposed a method for depositing superconductive thin film on a substrate using repetitive impulse discharges. (") In this method, however, effects of the impulse discharge on emission and deposition, and characteristics of the species emitted due to the discharges are not yet enough clarified. Therefore, they have to be probed continuously and in detail in order to establish the thin film deposition method.

In that connection, the authors already measured the axial distribution of gas temperature and its temporal variation in the impulse discharge. (s) Both the relative intensity distribution and the line intensity ratio of rotational spectra of the 2nd positive $(0,1)$ band of $N_{2}$ were used. In these methods for measurement of gas temperature, however, the measurable region is limited to the discharge channel by reason of depending on the optical emission from the channel.

So, this study aims to reveal the gas dynamic phenomena with a high accuracy in a wide gap space extended to the outside of the discharge channel by using laser scattering techniques. The spatiotemporal distributions of gas temperature were measured using multiplex coherent anti-Stokes Raman spectroscopy (CARS), the electron density using Thomson scattering, and the density of $\mathrm{Cu}$ atoms from the electrodes using laser induced fluorescence (LIF). These measurements provide the local values with a spatial resolution less than $0.1 \mathrm{~mm}$ determined by the laser beam divergence, and a temporal resolution of several ns determined by the laser pulse width.

\section{Observation of optical emission process}

Figure 1 shows the circuit for generating impulse discharge and the measurement system of optical emission process and electric signals. The needle to plate electrodes having a protrusion on the plate were used here. The gap length between the tips of the needle and protrusion was $20 \mathrm{~mm}$, and the discharge chamber was filled with room air of $66.7 \mathrm{hPa}$ in pressure.

A positive impulse voltage of $4.0 \mathrm{kV}(1 / 300 \mu \mathrm{s})$ was 
applied to the needle electrode, which generated the impulse glow-arc discharge. The framing photograph of the gas discharge was recorded by means of an image converter camera, and this was used for identification of the discharge processes and for discussion of the behaviors of the discharge channel. Along with observation of the optical emission process of the gas discharge, the voltage over the gap and the discharge current during the impulse discharge were measured by using a digital oscilloscope.

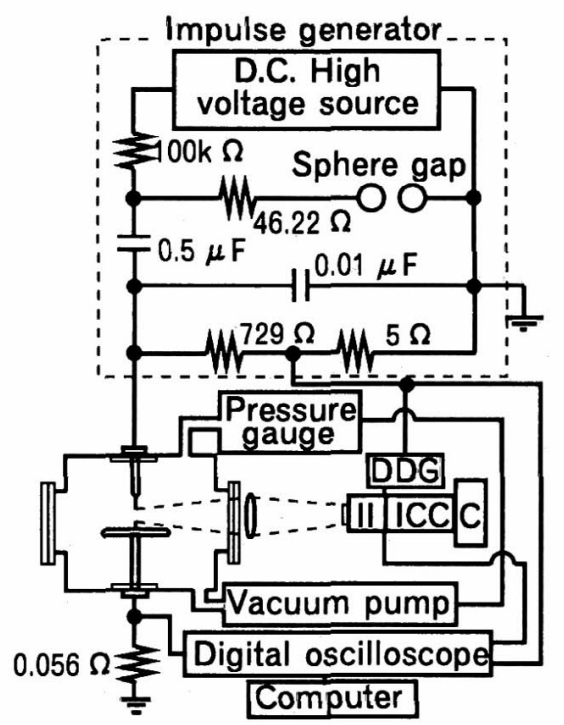

C: Camera II: Image intensifier ICC: Image converter camera DDG: Digital delay generator

Fig.1. Measurement system of optical emission

Figure 2 shows (a) the framing photographs, (b) the sketches of them and (c) the waveforms of applied voltage and discharge current of an impulse discharge which turns to arc from transitional glow. In the sketches, the bright regions in the framing photographs are represented by dense dots, and dark regions by sparse dots.

From these framing photographs and the current waveform, it is found that the instantaneous value of current increases as two constricted parts of the positive column (CPCs) ${ }^{(5)}$ extend, and rises rapidly with the occurrence of arc when the glow to arc transition appears at $1.8 \mu \mathrm{s}$ with the positive column still having a diffused part (DPC) ${ }^{(5)}$. The arc is initiated by the establishment of a cathode spot. Also, it seems that the constriction in the glow stage causes the increases of current density and heating of neutrals, and affects the transition to arc. The discharge current reaches a peak value of about $110 \mathrm{~A}$ at about $2.3 \mu \mathrm{s}$ when two CPCs combine at the middle of the gap.

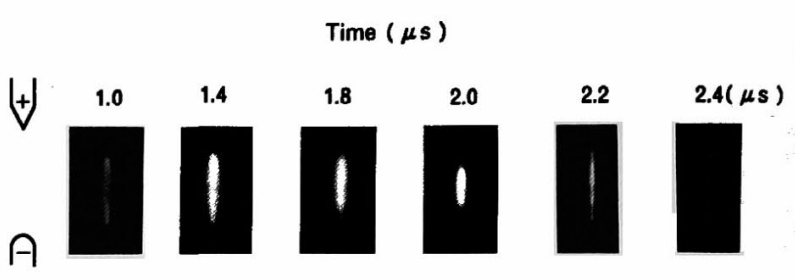

(a) Framing photographs

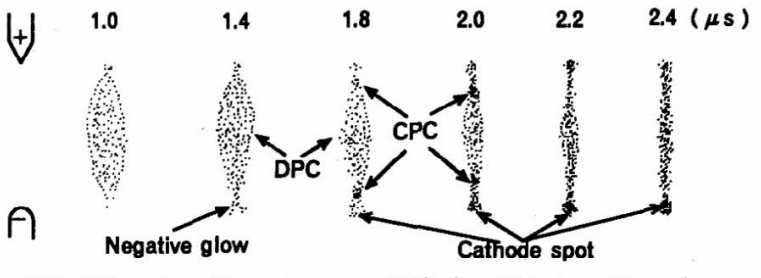

DPC: Diffused positive column CPC: Constricted positive column

(b) Sketches of framing photographs

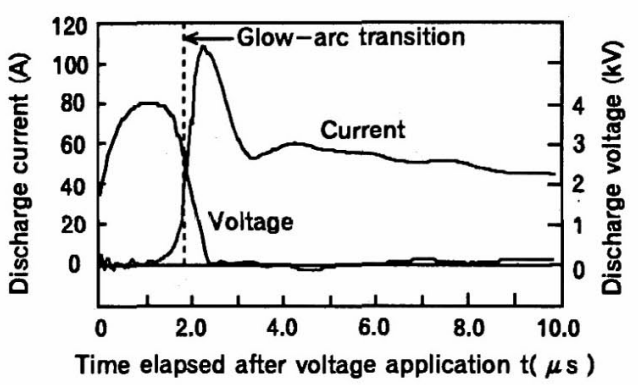

(c) Discharge voltage and current

Fig.2. (a)Framing photographs, (b)sketches of framing photographs and (c)waveforms of discharge voltage and current

3. Measurements of discharge dynamics using laser scattering

3.1 Multiplex CARS for the gas temperature measurement

CARS is one of non-linear Raman scattering techniques characterized by the coherent generation of Raman radiation based on a third order non-linear susceptibility. (6) CARS consists of the excitation of a Raman-active molecular vibration medium by a pair of high power laser beams. When the difference between angular frequencies $\omega_{1}$ and $\omega_{2}$ of the polarized laser beams is close to a Raman active angular frequency $\omega \mathrm{r}$ of molecules, the molecular polarization with a frequency of $\omega_{3}=2 \omega_{1}-\omega_{2}$ builds up collectively.

Multiplex CARS which uses the beam of $\omega_{2}$ with a wider linewidth than that of $\omega$, can obtain the broadband CARS spectra. As multiplex CARS can obtain the CARS spectra by only one laser shot, this technique suits the measurement of gas temperature in a transient phenomenon like an impulse discharge.

The gas temperature is determined from comparing the observed CARS spectra with the simulated ones which are 
caluculated by considering the third order non-linear susceptibility. (") The third order non-linear susceptibility is calculated by the spectral profiles of laser beams, the rotational and vibrational structure in nitrogen molecules, and the distribution function. In this study, the intensity distribution of CARS spectra of Q-branch was calculated.

By observing the Q-branch CARS spectra of nitrogen molecules in thermal equilibrium, we can obtain a rotational temperature. The rotational temperature is considered to agree with the gas temperature because the relaxation time of transition between the rotational and the translational energies in an air pressure of $66.7 \mathrm{hPa}$ is less than $10 \mathrm{~ns}$ by estimating in consideration of collisional frequency of $\mathrm{N}_{2}$ and $\mathrm{O}_{2}$. Then, the measurement of the order of $\mu \mathrm{s}$ is done in thermal equilibrium. We can determine the gas temperature within an accuracy of $\pm 10 \%$ at the room temperature in this technique.

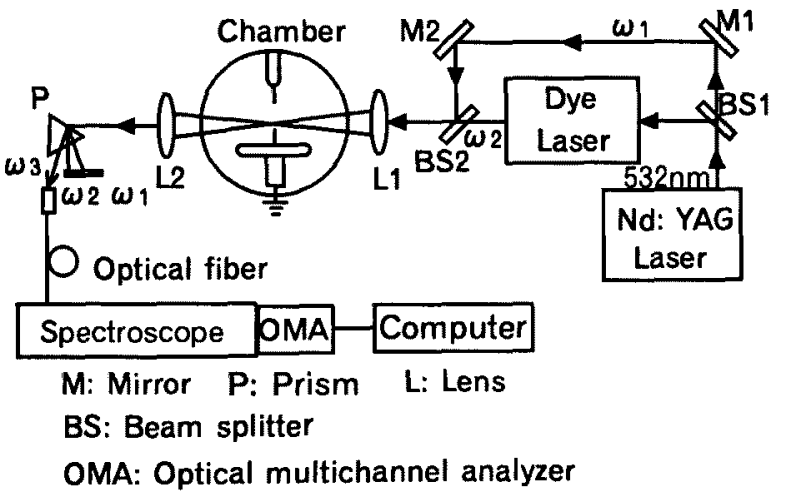

Fig.3. Schematic diagram of multiplex CARS system

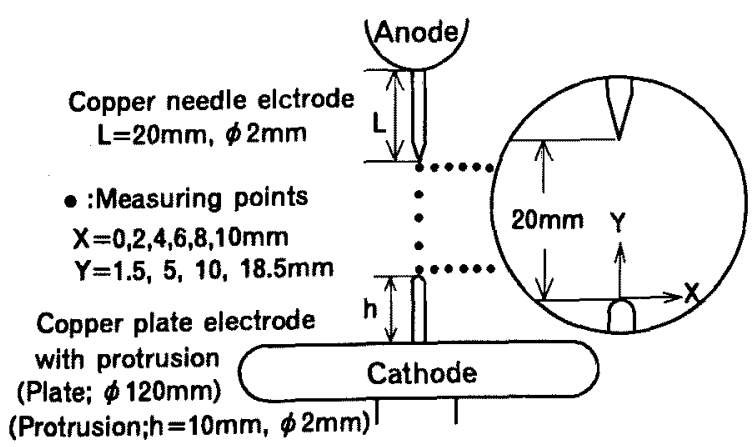

Fig.4. Configuration of electrode system and measuring points

Figure 3 shows a schematic diagram of the multiplex CARS system used for observation of the CARS spectra which are $Q$-branch rotational ones of $\mathrm{N}_{2}(0,1)$ vibrational band. The $\omega$, beam had an energy of $65 \mathrm{~mJ}$ at $532 \mathrm{~nm}$ (linewidth $1.0 \mathrm{~cm}^{-1}$ ) of a frequency-doubled Nd:YAG laser. The pulse duration of the laser was about $7 \mathrm{~ns}$. The $\omega_{2}$ was tuned to a wavelength of $607.3 \mathrm{~nm}$ (linewidth $80 \mathrm{~cm}^{-1}$ ) of a dye laser with an energy of $8 \mathrm{~mJ}$ pumped by the laser mentioned above. CARS spectra of $\omega_{\text {a }}$ were observed using a spectroscope with an optical multichannel analyzer.

Figure 4 shows a schematic diagram of the configuration of needle to plate electrodes having a protrusion on the plate and the measuring points of the impulse discharge. The gas temperatures were measured at the points of $\mathrm{X}=0$ (inside the channel) and $X=2,4,6,8,10 \mathrm{~mm}$ (outside the channel) keeping the height constant at each of $\mathrm{Y}=1.5,5$, $10,15,18.5 \mathrm{~mm}$ from the tip of the protrusion.

\subsection{LIF for the measurement of $\mathrm{Cu}$ atom density}

The experimental setup for the measurement of $\mathrm{Cu}$ density by LIF (a) is shown in Fig. 5 . It consists of a Nd: YAG laser pumped dye laser, a gas discharge chamber and a detection system. In the detection system, a photomultiplier tube is used for sensitive detection.

For the measurement of the density of $\mathrm{Cu}$ atoms from the electrodes, a two-level system for laser excitation and fluorescence detection at $324.75 \mathrm{~nm}\left(4 \mathrm{~s}^{2} \mathrm{~S}_{1 / 2} \leftarrow 4 \mathrm{p}^{2} \mathrm{P}^{0} 1 / 2\right)$ was used. The peak power of the dye laser through a filter was $178 \mathrm{~W}$ at $324.75 \mathrm{~nm}$ with a methanol solution of DCM 640 dye. This value was much higher than the saturation power $(0.3 \mathrm{~mW}$ at $324.75 \mathrm{~nm})$ calculated in consideration of a spectral width of $0.005 \mathrm{~nm}$ and a condensed laser cross section of $4.38 \times 10^{-10} \mathrm{~m}^{-2}$. The dye laser beam was introduced into a measuring point and focused by a $400 \mathrm{~mm}$ focal length lens. The LIF signal was observed from the direction normal to the laser beam, through a collecting lens, an optical fiber, a spectroscope and a photomultiplier tube.

As the fluorescence intensity is proportional to the atomic density, the absolute density can be calibrated by the Rayleigh scattering (a) from a gas with known scattering cross section at room temperature. In this experiment, 66.7hPa of Ar gas was used for that calibration. Here, we neglected the quenching by the collision between $\mathrm{Cu}$ atoms and the low pressure air.

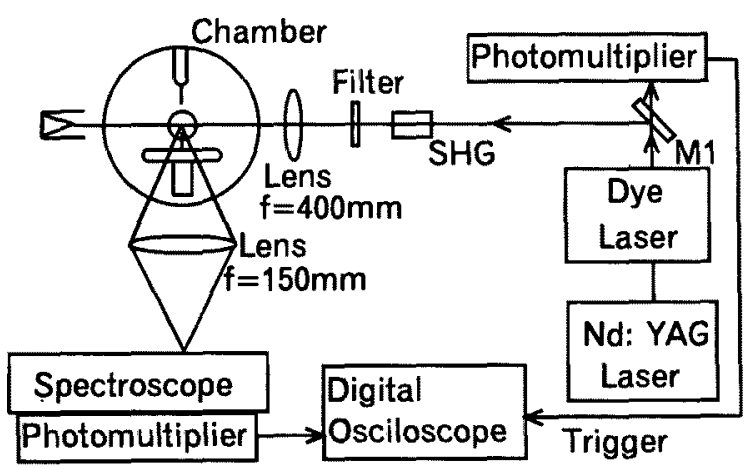

Fig.5. Schematic diagram of LIF system 


\subsection{Thomson scattering for the measurement of electron} density

In order to understand the internal mechanisms of discharge plasmas, it is important to clarify the electron behavior because the electrons are initially energized by the discharge heating process and then induce physical and chemical reactions upon other plasma species.

Laser Thomson scattering provides local measurements of electron density and possesses few ambiguities in the interpretation of data. (3) (9) When the scattering parameter $(0)$ is over 1 , the scattering is called the "collective scattering". This scattering consists of the contribution of electrons and ions. The spectral width of ion term is much narrower than that of electron term and the scattered power in the electron and ion terms are the same order of magnitude. "10) Then, the measurements were done on the ion term because this scattered emission power per unit wavelength is large.

The experimental setup for the measurement of electron density is almost similar to that of LIF. The second harmonic beam from a Nd:YAG laser, with a wavelength of $532 \mathrm{~nm}$, was used as a radiation source for the Thomson scattering experiment. The laser had a pulse energy of $150 \mathrm{~mJ}$ and a pulse duration of $7 \mathrm{~ns}$. Combined with Rayleigh scattering from an Ar gas for the same optical system, the absolute electron density is calibrated within an accuracy of $\pm 15 \%$. "10)

\section{Experimental results and disscusions}

4.1 Gas temperature, electron density and density of $\mathrm{Cu}$ in a discharge channel

The gas temperature and electron density which were obtained near the tips of anode $(X=0 \mathrm{~mm}, Y=18.5 \mathrm{~mm}$ ) and cathode $(X=0 \mathrm{~mm}, \mathrm{Y}=1.5 \mathrm{~mm})$ of the impulse discharge are shown in Figs. 6 and 7 . In these figures, the error bars indicate the range of scatter in five different measurements. Both of the gas temperatures obtained near the tips of anode and cathode go up suddenly at the transition from glow to arc, and come up to the highest at the peak of current. The peak values of gas temperature near the tips of anode and cathode are $4800 \mathrm{~K}, 4300 \mathrm{~K}$, respectively.

In this stage, the electrons having high density are emitted from the cathode by the growth of arc and the combination of the two proceeding CPCs shown in Fig.2. These phenomena are proved in the variation of the electron density in Fig.7. Both of the electron densities obtained near the tips of anode and cathode behave as same as the variations of gas temperature in the process of initial arc. Therefore, the electrons elevate remarkably the gas temperature near the anode and cathode.
After that discharge process, however, the gas temperature become low, since the stored charge in the electrostatic capacity of the power circuit becomes insufficient. That is, although the gas temperature increases with the discharge current in the beginning, it decreases in keeping with the current, which decreases with the rest of the charge stored in the capacitor. However, the gas temperature near the tip of cathode keeps a little higher than that near the tip of anode since the cathode spot keeps longer than the anode spot. (5)

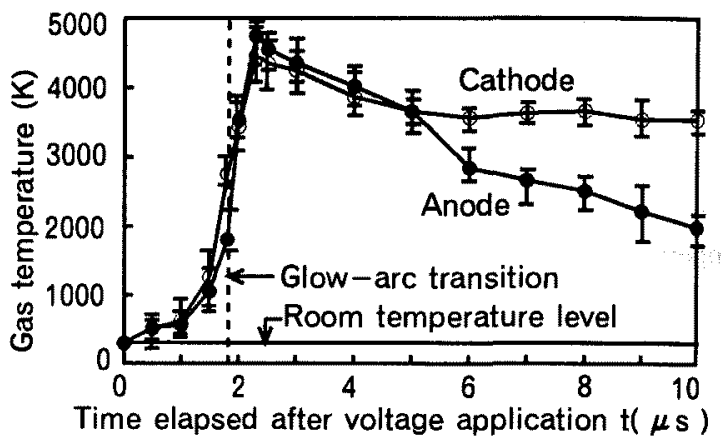

Fig.6. Gas temperature obtained at near the tips of anode and cathode

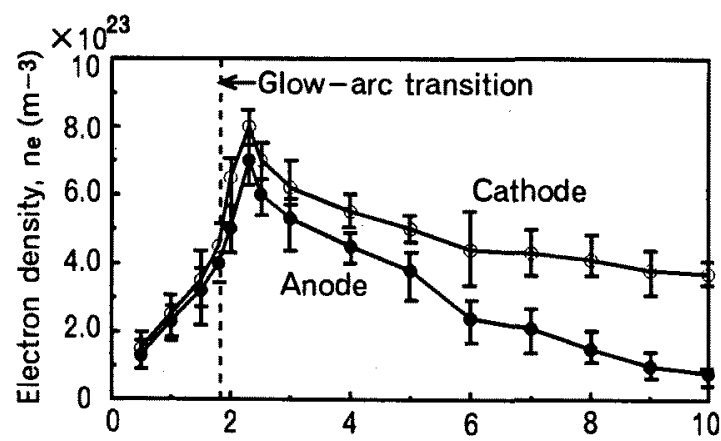

Time elapsed after voltage application $t(\mu s)$

Fig.7. Electron density obtained near the tips of anode and cathode

Figure 8 shows the spatiotemporal distribution of gas temperature obtained along the discharge channel. In this figure, the broken line shows the development of the tips of CPCs refered to the results of framing photographs.

The gas temperature of discharge channel is quite low when the discharge phase is diffused glow. However, the gas temperature increases with CPC's proceeding, and of the CPC is higher than that of the DPC at the transitional glow discharge phase. After the transition from glow to arc, both gas temperatures near the anode and cathode are higher than that of the arc column. This phenomenon is probably caused by the higher electron density near the anode and cathode.

Figure 9 shows the variations of $\mathrm{Cu}$ atomic density observed near the tips of anode ( $X=0 \mathrm{~mm}, Y=18.5 \mathrm{~mm}$ ) and cathode ( $X=0 \mathrm{~mm}, Y=1.5 \mathrm{~mm}$ ). From this figure, 
it is found that $\mathrm{Cu}$ atoms appears even in the transitional glow process. The density of $\mathrm{Cu}$ atoms measured near the cathode in this transitional glow stage is higher than that near the anode. The gas temperature near the cathode was also $2700 \mathrm{~K}$ high at the transitional glow which is higher than the melting point of $\mathrm{Cu}$ metal; that of the anode was about $1700 \mathrm{~K}$.

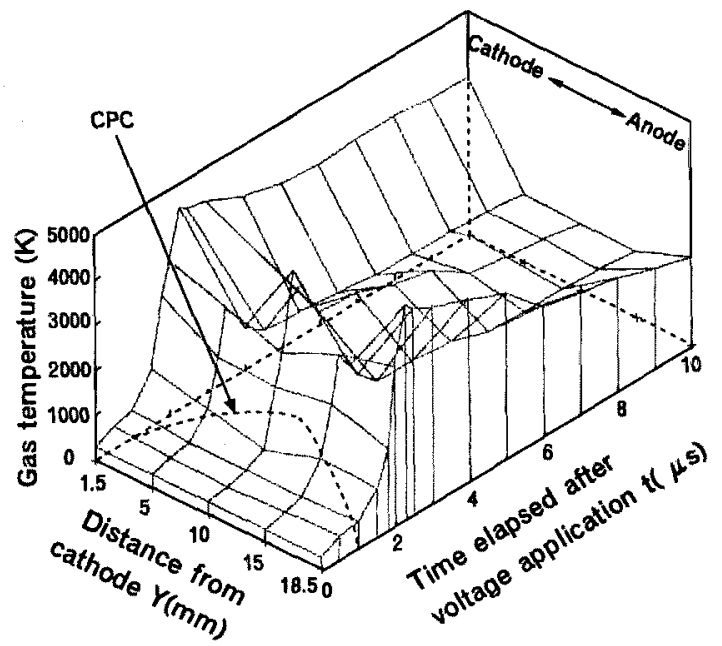

Fig.8. Distribution of gas temperature along the gap axis

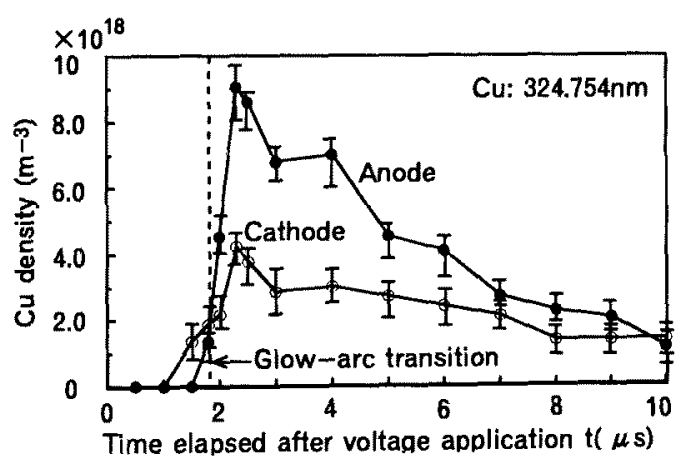

Fig.9. Variations of $\mathrm{Cu}$ atoms density near the tips of the anode and cathode

Accodingly, the measured $\mathrm{Cu}$ atomic density before the tansition from glow to arc seems to depend on evaporation and sputtering due to the high electric field and the high temperature near the cathode surface. The electric field near the electrodes seems to become higher with the development of CPC and an increase in discharge current. Here, the low ionization potential of $\mathrm{Cu}$ will also have an effect on the above result.

In the initial arc process, however, the density of $\mathrm{Cu}$ atoms near the anode becomes larger than that near the cathode, and the peak value near the anode reaches about 2 times as high as that near the cathode; whereas the peak gas temperature near the anode was only a little higher than that near the cathode. This phenomenon is believed to be related to the energy injection mechanism of electrons into the anode or to the ionization / excitation of $\mathrm{Cu}$ atoms near the cathode. Further measurements are needed in order to investigate this phenomenon.

\subsection{Gas temperature and $\mathrm{Cu}$ density near the discharge channel}

We measured the spatiotemporal distributions of the gas temperature and the density of $\mathrm{Cu}$ atoms at the measuring points of $X$ direction near the anode $(Y=18.5 \mathrm{~mm})$ and cathode $(Y=1.5 \mathrm{~mm})$ in Fig. 4 . It was found that both peaks of the gas temperature and density of $\mathrm{Cu}$ atoms at a measuring point outside the channel appear later than those in the channel as shown in Fig. 10.

Figure 11 shows the velocity of individual peaks from the inside to the outside of the channel. It is found that each moving velocity of peaks of gas temperatures and $\mathrm{Cu}$ density changes in the same manner "'" and moves quickly from the inside toward the outside of the channel; their moving velocities decreased from $1200 \mathrm{~m} / \mathrm{s}$ to $200 \mathrm{~m} / \mathrm{s}$ within $20 \mu \mathrm{s}$.

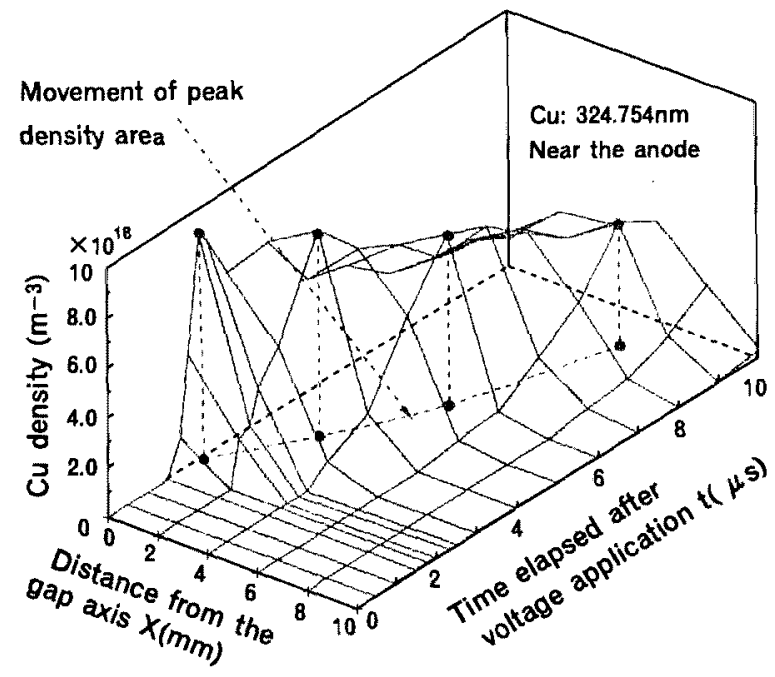

Fig.10. Density of $\mathrm{Cu}$ near the anode

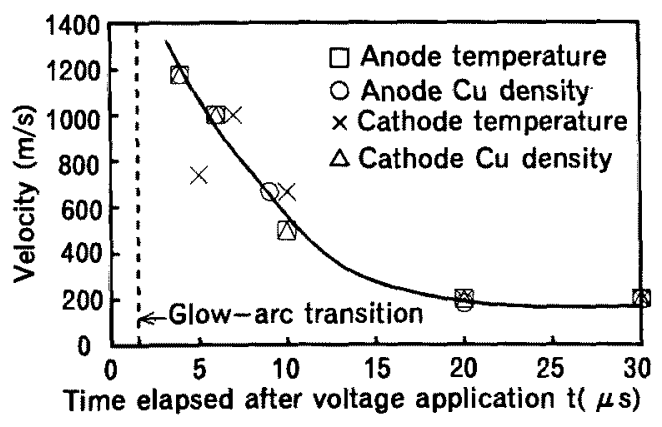

Fig.11. Velocities of peak values of gas temperature and $\mathrm{Cu}$ density 


\section{Conclusion}

We applied the laser scattering techniques to the diagnostics of the impulse discharge in the low pressure air. The gas temperature was measured using multiplex CARS, electron density using Thomson scattering and the density of $\mathrm{Cu}$ atoms from the electrodes using LIF. The results are summarized as follows.

(1) The gas temperature was higher in the constricted regions, especially at the two parts near the tips of anode and cathode along the positive column.

(2) The gas temperature obtained near the tips of anode and cathode was higher than melting point of $\mathrm{Cu}$ before the transition from glow to arc, and the sputtered and evaporated $\mathrm{Cu}$ atoms were measured.

(3) The gas temperature and the density of $\mathrm{Cu}$ atoms obtained near the tips of anode and cathode became high with a rise of discharge current and at the transiton from glow to arc, and highest when the discharge current took a peak value as same as the electron density.

(4) The density of $\mathrm{Cu}$ atoms obtained near the tip of anode was about 2 times as high as that near the tip of cathode.

(5) The spatial evolution of the gas temperatures and $\mathrm{Cu}$ density outside the discharge channel had occurred with the same characteristis.

(Manuscript received January 19, '96, revised June 4, '96 )

\section{References}

(1) M. Baba, T. Sakoda, K. Nakao, M. Otsubo, H. Nieda "Gas Temperature Measurement in DC Glow and Impulse Discharge by Multiplex CARS Method" Proc. 8th Int. Symp. on High Voltage Engineering, pp.387-390, 1993

(2) T. Sakoda, M. Otsubo, C. Honda, H. Nieda, "Gas Temperature and $\mathrm{Cu}$ Vapor Measurements in and near an Impulse Discharge by Multiplex CARS and LIF Method" Proc. 7th Asian Conf. on Electrical Discharge, pp.196-199, 1994

(3) K. Uchino, Y. Kubo, K. Muraoka, T. Sakoda, H. Yamakoshi, M. Kato, A. Takahashi, M. Maeda, "Investigation of Electron Density and Velocity Distribution in High - Pressure Discharges for Excimer Laser Pumping using Laser Thomson Scattering" J. Appl. Phys., Vol.70, No.1, pp.41-45, 1991

(4) T. Komura, T. Yamaguchi, M. Otsubo, T. Matsune, H.Nieda, "Spectroscopic Observation and Temperature Characteristics of the Plasma in Preparation Process of Thin Film by Impulse Discharges" Proc. 6th Asian Conf. on Electrical Discharge, pp.281-284, 1993

(5) M. Otsubo, H. Nieda, H. Matsuo, "Gas Temperatures in Impulse Discharge Channels at Low-Pressure Air" Jpn. J. Appl. Phys., Vol.31, No.3, pp.887-895, 1992

(6) S. Maeda, T. Kamisuki and Y. Adachi, "CONDENSED PHASE CARS" in Advances in Non-linear Spectroscopy, Chap. 6, Ed. R. J. H. Clark and R. E. Hester, John Wiley \& Sons Ltd., 1988

(7) M. Kawasaki, E. Kawai, H. Sato, K. Sugai and M. Hanabusa, "CARS Spectroscopy of $\mathrm{SiH}_{4}$ and $\mathrm{Si}_{2} \mathrm{H}_{6}$ in Supersonic Free Jets" Jpn. J. Appl. Phys., Vol.26, No.9, pp.1395-1399, 1987

(8) Jerry A. Gelbwachs, "Impurity Monitoring by LaserInduced Fluorescence Techniques" Rev. Sci. Instrum., Vol.56, No.5, pp.1000-1004, 1985

(9) M. D. Bowden, F. Kimura, H. Muta, K. Uchino, K. Muraoka and M. Maeda,"Thomson Scattering Measurements of Electron Temperature and Density in an Electron Cyclotron Resonance Plasma" J. Appl. Phys., Vol.73, No.6, pp.2732-2738, 1993

(10) K. Uchino, K. Muraoka and M. Akazaki,"Laser Scattering Diagnostics of an Atmospheric Impulse Arc", Jpn. J. Appl. Phys., Vol.20, No.6, pp.1165-1166, 1981

(11) T. Sakoda, M. Otsubo, C. Honda, H. Nieda, "Measurements of Gas Temperature and $\mathrm{Cu}$ Vapor Density in Lighting Impulse Discharge using Multiplex CARS and Laser Induced Fluorescence Spectroscopy" Proc. 9th Int. Symp. on High Voltage Engineering, pp.2151-1 - 2151-4, 1995

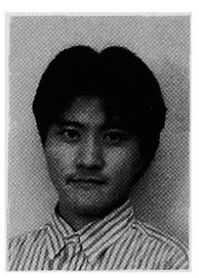

Tatsuya Sakoda (student member) He was bom in 1970. He received his B.E. and M.E. degrees in electrical engineering from Miyazaki University in 1993 and 1995 respectively. Since 1995, he is a doctor course student at Kyushu University. His research interests include the plasma engineering phenomena.

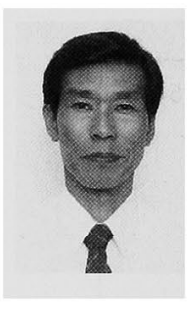

Masahisa Otsubo (member) He was bom in 1947. He received his B.E. degree in electrical engineering from Miyazaki University in 1969, and his Dr. degree from Kumamoto University in 1993. He was engaged as a Research Associate in Miyazaki University in 1970, and has been an Associate Professor since 1993. His research interests include the dielectric insulation of polymers and the discharge plasma phenomena.

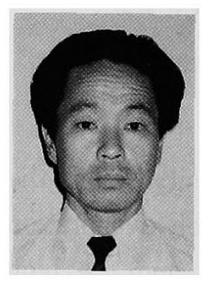

Chikahisa Honda (member) He was born in 1943. He received his B.E. and Dr. degrees in electrical engineering from Kyushu University in 1965 and 1988 respectively. He was engaged as a Research Associate and Associate Professor in Kyushu University in 1965 and 1989 respectively, and has been a Professor in Miyazaki University since 1993. He has been engaged in research of plasma diagnostics.

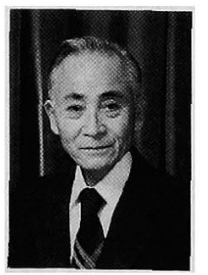

Hiroshi Nieda (member) He was borm in 1927. He was engaged a Research Associate, Assaciate Professor and Professor in Miyazaki University in 1963, 1968 and 1986 respectively. He has been a Professor in Kumamoto Institute of Technology since 1993 and engaged in research of high voltage engineering and discharge plasma phenomena. 